\title{
COVID-19: Wie änderte sich die Sterblichkeit? - Mortalität von Frauen und Männern in Deutschland und seinen Bundesländern bis Oktober 2020
}

\section{COVID-19: How did mortality change? - Mortality of women and men in Germany and its federal states until October 2020}

„Was kommt auf uns zu“ [1] fragten Kern et al. mit Bezug auf offene Fragen zur Sterblichkeit im Zusammenhang mit SARS-CoV-2-Infektionen. Um bei der Bewältigung der Pandemie „mit viel Sicht fliegen“ " 2,3$]$ zu können, sollten in SARSCoV-2/COVID-19-Zeiten Virus-Testergebnisse um epidemiologische Ergebnisse zur Sterblichkeit ergänzt werden. Auswertungen der Mortalität für Frauen und Männer, die das Alter berücksichtigen, und mit angemessener räumlicher Auflösung erfolgen [4], d. h. in den 16 Bundesländern, bieten wichtige Informationen.

\section{Methoden}

Wir analysierten die Gesamtmortalität, getrennt für Kombinationen von Geschlecht (gesamt, männlich, weiblich) und Alter (gesamt, $<65, \geq 65$ Jahre), für Deutschland und seine Bundesländer für den Zeitraum Januar bis Oktober in den
Jahren 2016-2020 auf Basis der aktuellen Daten des Statistischen Bundesamtes (veröffentlicht am 27.11.2020, im Supplement enthalten). Als Effektmaße berechneten wir vereinfachte Standardisierte Mortalitätsratios [5] [SMRs] mit erweiterten $95 \%$-Konfidenzintervallen ( $95 \% \mathrm{KI}$, erweitert $=$ random-effects-Ansatz [6]) pro Woche und Monat als das Verhältnis aus der Zahl der Todesfälle im Jahr 2020 und einem Mittelwert (arithmetisch bzw. geometrisch) der Zahl der Todesfälle in den Referenzjahren 2016 bis 2019. Details zur Methodik inkl. Auswerteprogramm finden sich online im Supplementary Material.

\section{Ergebnisse}

Von Januar bis Oktober betrug die Gesamt-SMR (geometrisch) für Deutschland 1,01 (95\% KI: 0,99-1,04). Die GesamtSMR für Frauen war 1,00 (95\% KI: 0,981,03 ) und für Männer - statistisch signifi- kant - erhöht 1,03 (95\% KI: 1,01-1,06). Für Frauen $<65$ bzw. $\geq 65$ Jahren wurden eine geringe Untersterblichkeit - statistisch signifikant - bzw. Übersterblichkeit bestimmt (<65 Jahre: SMR $=0,98 ; 95 \% \mathrm{KI}$ : 0,96-0,99; $\geq 65$ Jahre: SMR $=1,01 ; 95 \% \mathrm{KI}$ : 0,98-1,04). Bis 65-jährige Männer hatten insgesamt keine erhöhte Sterblichkeit; in der Altersgruppe $\geq 65$ Jahre zeigte sich eine erhöhte SMR - statistisch signifikant - von 1,04 (95\% KI: 1,01-1,08).

- Abb. 1 ist bis Ende Oktober mit zwei Phasen von Exzess-Mortalitäten vereinbar, eine im April/Mai und eine im August/September. Darüber hinaus zeigt die Grafik, dass über 10 Monate insgesamt erhöhte SMRs bei Frauen in 4 und bei Männern in 12 Bundesländern beobachtet wurden: In Niedersachsen, Baden-Württemberg, Bayern, Berlin und Brandenburg waren die Übersterblichkeiten bei Männern statistisch signifikant. Für mindestens 65-jährige Männer wurden z. T. ausgeprägte Exzess-Morta-
Deutschland

Schleswig-Holstein

Hamburg

Niedersachsen

Bremen

Nordrhein-Westfalen

Hessen

Rheinland-Pfalz

Baden-Württemberg

Bayern

Saarland

Berlin

Brandenburg

Meckl.-Vorpommern

Sachsen

Sachsen-Anhalt

Thüringen
Frauen

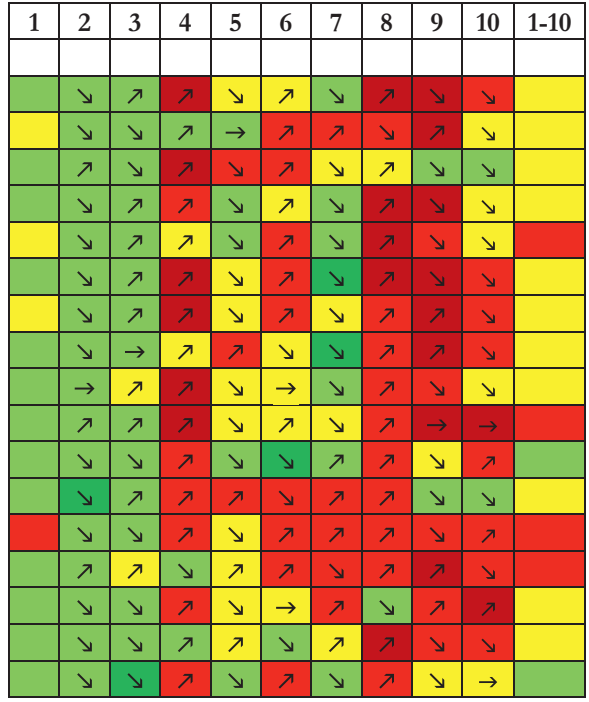

Männer

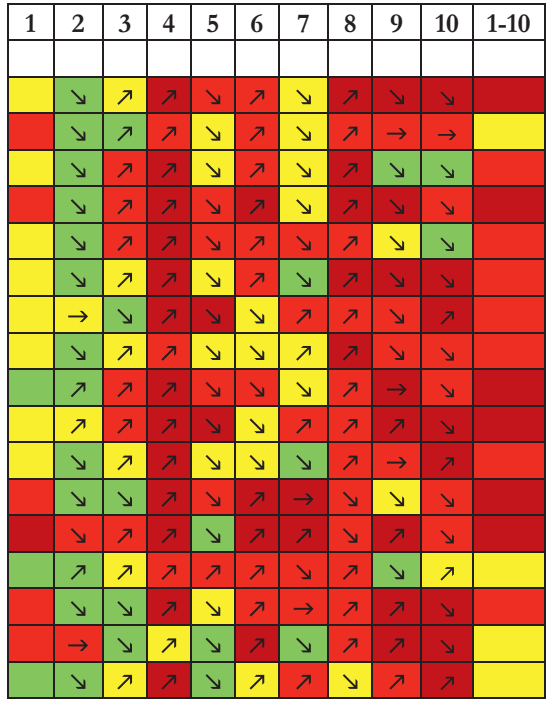

- Abb. 1 Relative Sterblichkeit (SMR, geometrisch) an allen Todesursachen für Frauen und für Männer in Deutschland und in den 16 Bundesländern von Januar (1) bis Oktober (10) 2020 im Vergleich zu 2016-2019 und für den gesamten Zeitraum (1-10). Dunkelgrün: SMR < 1 und 95\% KI schließt die 1 aus; Hellgrün: SMR < 0,99 und $95 \%$ KI schließt die 1 ein; Gelb: SMR = 0,99-1,01 und $95 \%$ KI schließt die 1 ein; Hellrot: SMR > 1,01 und $95 \%$ KI schließt die 1 ein; Dunkelrot: SMR > 1,0 und $95 \%$ KI schließt die 1 aus; Pfeile zeigen an, ob SMRs im Vergleich zum Vormonat gestiegen, gesunken oder gleich geblieben sind. 


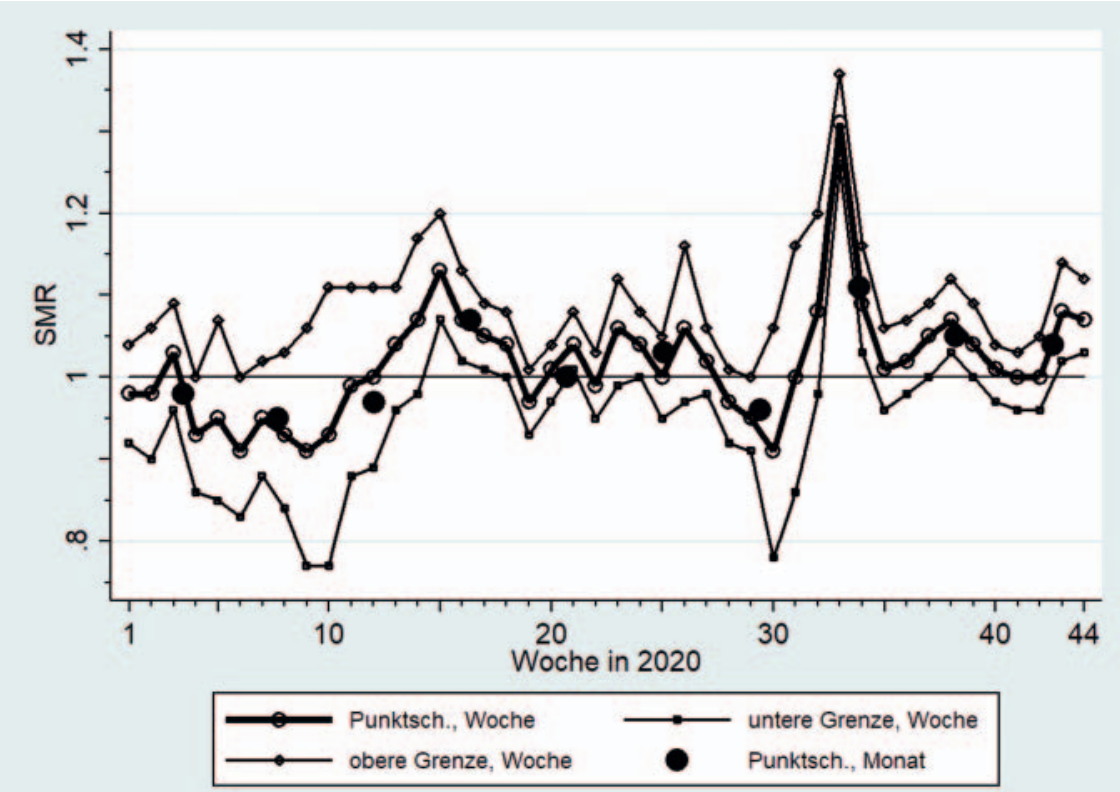

- Abb. 2 Relative Sterblichkeit (SMR-Punktschätzer mit $95 \%$-KI, geometrisch) in NordrheinWestfalen für Frauen und Männer gemeinsam: Mortalität an allen Todesursachen von Januar bis Oktober 2020 im Vergleich zur Mortalität 2016-2019.

litäten in Deutschland sowie allen Bundesländern bestimmt. > Abb. 2 zeigt beispielhaft den Verlauf der SMR in Wochenauflösung, der der Zeile Nordrhein-Westfalen in

- Abb. 1 zugrunde liegt.

Mecklenburg-Vorpommern hatte eine lange Periode (Januar bis April) von SMRs unter oder nahe 1, obwohl die SMRs anstiegen - seit Mai/Juni auch mit Übersterblichkeiten. Geschätzte Trends in Mecklenburg-Vorpommern zeigten bis Juni einen SMR-Anstieg von 2,5\% pro Monat oder 4 Wochen, der auch nach Adjustierung für Alter und Geschlecht beobachtet wurde (immer $p<0,0005$ ).

Die Fallzahlen, SMRs und Konfidenzintervalle in Tabellenform, differenziert nach Alter und Geschlecht, pro Monat und Woche sowie gesamt bis Oktober 2020 zu Deutschland und zu allen Bundesländern sind als Supplementary Material online verfügbar.

\section{Diskussion}

Trotz der offensichtlichen Erhöhungen der Sterblichkeit im April/Mai in Assoziation mit COVID-19 und im August in Koinzidenz mit einer Hitzewelle [7], entsprach in Deutschland die Mortalität von Januar bis Oktober im Jahr 2020 beinahe genau dem Durchschnittswert der vom Statistischen Bundesamt angebotenen Referenzjahre 2016 bis 2019 (in denen sich Grippeund Hitzewellen ereigneten). Dies bestätigt Befunde von Stang et al. [8]. Übereinstimmend mit [9] ergaben unsere Analysen eine für Männer erhöhte und für beide Geschlechter mit dem Alter ansteigende relative Mortalität in 2020 (vgl. zu Limitationen die Ausführungen im Supplement). Auch bei Personen < 65 Jahre sahen wir einen gewissen SMR-Anstieg im April/ Mai, aber insbesondere auch im Zeitfenster der Hitzewelle im August [7].

Das Beispiel von Mecklenburg-Vorpommern kann veranschaulichen, wie Mortalitätsanalysen Virustests ergänzen können und sollten [2, 3]: Bis April 2020 war die Mortalität dort nicht erhöht, wenn sie anhand der SMR der Einzelmonate beurteilt wurde, und der SMR-Anstieg von März bis April war gering. Bei vergleichsweise wenig dokumentieren Infektionen schlug die dortige Landesregierung Anfang Juli Gespräche zur Abschaffung der Maskenpflicht im Handel vor. Die Untersuchung der SMRs von Januar bis Juni zeigte für Mecklenburg-Vorpommern jedoch einen auffälligen linearen Trend zu steigenden SMRs, der zur Vorsicht hätte mahnen können.
Bisher publiziert das Statistische Bundesamt die Grunddaten mit einem Verzug von ca. 4 Wochen. Eine zeitnähere Bereitstellung dieser Informationen ist angezeigt, wie z. B. durch EuroMOMO (https:// www.euromomo.eu/about-us/contact/) realisiert. Deutschland nimmt an diesem europäischen Projekt mit lediglich zwei Bundesländern teil (Hessen, Berlin). Um das der COVID-19-Pandemie zuzuschreibende Mortalitätsgeschehen angemessen analysieren zu können, sollten die Sterbedaten differenziert nach Regionen, Geschlechtern, Altersgruppen und Todesursachen angeboten werden.

In der Gesamtschau bieten unsere Auswertungen - erstmalig - für Frauen und Männer getrennt, für unterschiedliche Altersgruppen und für die einzelnen Bundesländer für 10 Monate empirische Daten zu Änderungen der Sterblichkeit [1] während der SARS-CoV-2/COVID-19-Pandemie in Deutschland. Unter anderem aufgrund dessen, was in Italien, Spanien, Frankreich, dem UK und den USA durch überlastete Krankenhäuser dokumentiert wurde [10], kam es zu ausgeprägten Public-HealthMaßnahmen zur „Abflachung der epidemiologischen Kurve“ [10]. Zukünftige Forschungsarbeiten müssen klären, welche Bedeutung die ergriffenen Maßnahmen gegen COVID-19 für die derzeitigen Beobachtungen haben [11]. Der weitere Verlauf und die gesundheitlichen Auswirkungen der Pandemie bleiben zudem nicht klar vorhersehbar [10], da sich Effekte nicht notwendigerweise darauf beschränken, ob eine SARS-CoV-2-Infektion jetzt überlebt wird oder nicht. Aktuelle Sterblichkeitsdaten lassen z. B. Fragen zur Lebensqualität und Lebenserwartung offen, die sich zu potentiellen mittel- und langfristigen Folgen von COVID-19 ergeben können [12].

Zusätzliches Material finden Sie unter https://doi.org/10.1055/a-1334-0586

Interessenkonflikt

Die Autorinnen/Autoren geben an, dass kein Interessenkonflikt besteht. 
Danksagung

BT wird vom Koeln Fortune Program/Faculty of Medicine, University of Cologne unterstützt.

\section{Autorinnen/Autoren}

Peter Morfeld, Barbara Timmermann, Valérie J. Groß, Philip Lewis, Thomas C. Erren

Universität zu Köln, Institut und Poliklinik für Arbeitsmedizin, Umweltmedizin und Präventionsforschung, Uniklinik Köln

\section{Korrespondenzadresse}

\section{Dr. Peter Morfeld}

Universität zu Köln, Institut und Poliklinik für Arbeitsmedizin, Umweltmedizin und Präventionsforschung, Uniklinik Köln, Kerpenerstr. 62,

50937 Köln

Deutschland

peter.morfeld@ruhr-uni-bochum.de

\section{Literatur}

[1] Kern WV, Biever PM, Rieg S et al. [SARS-CoV-2 infection (COVID-19): what can we expect?]. Dtsch Med Wochenschr 2020; 145: 740-746. doi:10.1055/a-1114-3699
[2] Erren TC, Morfeld P. COVID-19-Mortalität: Mit viel Sicht fliegen. Dtsch Ärztebl 2020; 117: A-1010/B-850

[3] Morfeld P, Erren TC. Mortality and Attributable Fraction in COVID-19 Analysis: Avoiding Research Waste and Negligence. American Journal of Public Health 2020; 110: 16441645. doi:10.2105/AJPH.2020.305887

[4] Morfeld P, Timmermann B, Groß JV et al. COVID-19: Spatial resolution of excess mortality in Germany and Italy. I Infect 2020: S0163-4453(20)30678-2 doi:10.1016/j. jinf.2020.10.020

[5] Breslow NE, Day NE. Statistical methods in cancer research. Volume II - The design and analysis of cohort studies. Lyon: International Agency for Research on Cancer. 1987

[6] Sutton AJ, Abrams KR, Jones DR et al. Methods for Meta-analysis in Medical Research. Chichester, U.K: Wiley; 2000

[7] Robert-Koch-Institut. Täglicher Lagebericht des RKI zur Coronavirus-Krankheit-2019 (COVID-19). 30.10.2020 - Aktualisierter Stand für Deutschland; 2020

[8] Stang A, Standl F, Kowall B et al. Excess mortality due to COVID-19 in Germany. J Infect 2020; 81 (5): 797-801. doi:10.1016| j.jinf.2020.09.012

[9] Kremer H], Thurner W. Age dependence in Covid-19 mortality in Germany. Dtsch Arztebl Int 2020; 117: 432-433. doi:10.3238/ arztebl.2020.0432

[10] Muller O, Neuhann F, Razum O. [Epidemiology and control of COVID-19]. Dtsch Med Wochenschr 2020; 145 (10): 670-674. doi:10.1055/a-1162-1987
[11] Morfeld P, Erren TC. [Deaths in nine regions of Italy in February/March 2020: "Mortality Excess Loupe" for SARS-CoV-2/COVID-19Epidemiology in Germany]. Gesundheitswesen 2020; 82: 400-406. doi:10.1055/ a-1160-5859

[12] Yelin D, Wirtheim E, Vetter P et al. Long-term consequences of COVID-19: research needs. The Lancet. Infectious diseases 2020; 20: 1115-1117. doi:10.1016/S14733099(20)30701-5

\section{Publikationshinweis}

Leserbriefe stellen nicht unbedingt die Meinung von Herausgebern oder Verlag dar. Herausgeber und Verlag behalten sich vor, Leserbriefe nicht, gekürzt oder in Auszügen zu veröffentlichen.

\section{Bibliografie}

Dtsch Med Wochenschr 2021; 146: 129-131 Online-Publikation: 16.12.2020

DOI $10.1055 / \mathrm{a}-1334-0586$

ISSN 0012-0472

(C) 2020. Thieme. All rights reserved. Georg Thieme Verlag KG, Rüdigerstraße 14, 70469 Stuttgart, Germany 